\title{
Predicting taxi demand hotspots using automated Internet Search Queries
}

\author{
Markou, loulia; Kaiser, Kevin; Pereira, Francisco Camara
}

Published in:

Transportation Research. Part C: Emerging Technologies

Link to article, DOI:

10.1016/j.trc.2019.03.001

Publication date:

2019

Document Version

Peer reviewed version

Link back to DTU Orbit

Citation (APA):

Markou, I., Kaiser, K., \& Pereira, F. C. (2019). Predicting taxi demand hotspots using automated Internet Search Queries. Transportation Research. Part C: Emerging Technologies, 102, 73-86.

https://doi.org/10.1016/j.trc.2019.03.001

\section{General rights}

Copyright and moral rights for the publications made accessible in the public portal are retained by the authors and/or other copyright owners and it is a condition of accessing publications that users recognise and abide by the legal requirements associated with these rights.

- Users may download and print one copy of any publication from the public portal for the purpose of private study or research.

- You may not further distribute the material or use it for any profit-making activity or commercial gain

- You may freely distribute the URL identifying the publication in the public portal

If you believe that this document breaches copyright please contact us providing details, and we will remove access to the work immediately and investigate your claim. 


\title{
Predicting taxi demand hotspots using automated Internet Search Queries
}

\author{
Markou Ioulia*a $^{* a}$ Kevin Kaiser ${ }^{\mathrm{a}}$, Francisco C. Pereira ${ }^{\mathrm{a}}$ \\ ${ }^{a}$ Department of Management Engineering, Technical University of Denmark, Kongens Lyngby, Denmark
}

\begin{abstract}
Disruptions due to special events are a well-known challenge in transport operations, since the transport system is typically designed for habitual demand. Part of the problem relates to the difficulty in collecting comprehensive and reliable information early enough to prepare mitigation measures.

A tool that automatically scans the internet for events and predicts their impact would strongly support transport management in many cities in the world. This study addresses the challenges related to retrieving and analyzing web documents about real world events, and using them for demand explanation (if related to a past event) and prediction (if a future one).

Transport demand is predicted with a supervised topic modeling algorithm by utilizing information about social events retrieved using various strategies, which made use of search aggregation, natural language processing, and query expansion. It was found that a two-step process produced the highest accuracy for transport demand prediction, where different (but related) queries are used to retrieve an initial set of documents, and then, based on these documents, a final query is constructed that obtains the set of predictive documents. These are then used to model the most discriminating topics related to the transport demand. A framework was proposed that sequentially handles all stages of data gathering, enrichment, and prediction with the intention of generating automated search queries.
\end{abstract}

Keywords: demand prediction, special events, natural language processing, query expansion, information retrieval

\section{Introduction}

In general, mobility trends captured in complex transport systems consist of two basic components: utilitarian travel that mostly includes habitual behavior (e.g. commuting to work, weekly shopping) but also to a minor extent non-habitual needs (e.g. go to hospital,

\footnotetext{
*Corresponding author

Email addresses: markou@dtu.dk (Markou Ioulia*), mrkaikev@gmail.com (Kevin Kaiser), camara@dtu.dk (Francisco C. Pereira)
} 
occasional shopping); and recreational travel, which comprises the human need for entertainment, social interaction and public expression. Efficient and effective intelligent transport systems should be able to take into consideration both of these factors for accurate demand predictions and better traffic management.

Current prediction approaches generally focus on capturing recurrent conditions, namely their seasonal spatial-temporal aspects (the "average" winter peak-hour Monday, in area $\mathrm{X}$, with weather $\mathrm{Y}$ ). The developed approaches can be successful for long-term planning applications or for modeling demand in non-eventful areas such as residential neighborhoods. However, in lively and dynamic areas where multiple special events take place, such as music concerts, sports games, festivals, parades and protests, these approaches fail to accurately model mobility demand precisely at times when it is needed - when the transport system of the area is under stress. The inability of the system to meet the new demand conditions emphasizes the need of good anticipatory capabilities which are capable to accept timely information on such phenomena.

Non-recurrent special events, such as concerts, sport games and demonstrations, are planned and largely advertised on the Web. An interesting fact is that it is much more likely to have citizens sharing their expectations/experiences about non-recurrent events than to talk about their daily commute. This plethora of information makes the Web an important tool for demand prediction and thus systems balance maintenance.

Previous studies have shown a strong correlation between number of public transport arrivals with the structured data mined from the Web [1, 2]. Namely, semi-structured information about events from announcements websites can be used as features for public transport arrivals. It has also been highlighted that they can be incorporated into a prediction model using topic modeling [3] or embeddings [4] and significantly reduce the error in the forecasts. However, information contained on these websites is usually incomplete, noisy or missing, which makes it difficult to generalize. Going beyond this approach raises two new challenges: what web-pages are relevant (information retrieval) and how to turn relevant information into model attributes (information extraction).

The aim of this study is the exploitation of information available on the internet for future non-recurrent overcrowding (or hotspots) prediction. A particular emphasis will be given to special events that are publicly disclosed on social media and attract many people. The proposed framework will be able to correlate unstructured textual information with time-series traffic data with the ultimate goal of promptly alerting stakeholders for potential upcoming overcrowding alarms. It combines the Maximum entropy discriminant LDA (MedLDA) [5], a maximum margin supervised topic modeling algorithm, with textual data obtained from automatically generated queries. These queries are constructed from basic event information (title, location, time) obtained from event listing websites.

The paper is organized as follows. In the following sections we briefly review previous studies and discuss challenges related to traffic prediction, information retrieval and extraction. In sections 3 (Data Description and Preparation) and 4 (Methodology), we describe the proposed model and data used in its validation. Section 5 (Results) dedicates to analyze performance of the model. Possible limitations and extensions of the current investigation are summarized afterwards, in the Conclusion. 


\section{Literature Review}

The available spatial datasets, as well as the potential of events information and topic modeling for transportation problems, should be taken into consideration for an accurate demand prediction model formulation.

\subsection{Demand Prediction for special events}

Special events have a huge impact in urban mobility, regardless of their scale and type. Understanding their influence on the balance of a transport system is crucial for the development of reliable traffic management operations. For large-scale events (e.g. World cup, Formula One and Olympic games), best practices are already available for authorities to follow in order to manage these events and prepare for them well in advance $[6,7]$. However, these manual approaches do not scale to the vast amount of smaller and medium-sized events that take place on large metropolitan areas on a daily basis. Despite their reduced scale, these events still have a significant impact in the transportation system [2], especially when multiple co-occur. In these scenarios, common practice relies on reactive approaches rather than on planning $[8,9]$. The demand prediction solution that we propose in this paper, takes into consideration event information that is automatically mined from the Web, and present itself with the potential for anticipating the effects of events and showing reliable tools for hotspot predictions in eventful areas.

Traffic demand modeling can benefit to a significant extend from earlier stage predictions. Developed methodologies can be grouped into two general classes: disaggregate response, typically with discrete choice models, where individual behavior choices are represented as a function of individual's characteristics (e.g. gender, age) and alternative choice properties (e.g. cost, duration); and aggregate response, based on machine learning or classical statistics, where a response variable (e.g. travel delay, total attendance) is modeled as a function of available data (e.g. location, time of day, event category).

The research of [10] is included in the first framework class. They implemented an analysis of surveys conducted at three Turkish stadiums in advance and after matches, for the estimation of a binary logit model of mode choice (private car or public transport). A four-step model approach was proposed by $[9,11]$ to predict the number of trips, trip origin/destination, mode and vehicle miles traveled or transit boardings related to events. Despite being behaviorally sound and providing plenty of detail, these works highly depend on survey response and usually consider event features on a very superficial way. For instance, they rarely go deeper than general event category (e.g. sports, concerts).

The second framework includes several studies on forecasting demand. Some of the methods that have been proposed include Gaussian Processes [3], probabilistic graphical models [12, 13], neural networks [14] and time series modeling [15, 16]. Markou et al. [3] highlighted the importance of semantic information through the formulation of a real-time taxi demand prediction model. The incorporation of popular events' information using topic modeling resulted in a noticeable increase of forecasts' accuracy. Similar conclusions were presented through the implementation of two deep learning architectures that leverage word embeddings and convolutional layers for combining text information with time-series data 
by Rodrigues et al. [4]. A unified linear regression model that outperforms other popular non-linear models in the prediction accuracy is proposed by Tong et al. [17]. Particular emphasis was given to the conclusion that simple model structure that eliminates the need for repeated model redesign proves to be able to behave better in prediction scenarios with high-dimensional features. Finally, over the last decade, deep learning has enabled many practical applications of machine learning in the fields of transportation and urban mobility $[18,19,20]$.

Taxi demand has been the subject of several applications, since the related datasets are sufficiently detailed. The yellow and green taxi public dataset of New York City in particular, has been the subject of a lot of research. Morgul and Ozbay [21] present an empirical assessment of taxicab drivers labor supply. Yang and Gonzales [22] identify locations and times of day where there is a mismatch between the availability of taxicabs and taxi service demand. Zhao et al. [23] use entropy and the temporal correlation of human mobility to measure the demand uncertainty at the building block level. They implemented three prediction algorithms to validate their maximum predictability theory. We [24] used kernel density analysis for demand fluctuations detection and analysis. Significant deviations from the average day were correlated with disruptive event scenarios such as extreme weather conditions, public holidays, religious festivities, and parades. Finally, some other research studies used this taxicab data to explore taxicab drivers airport pick-up decisions [25], or travel time variability analysis [26].

\subsection{Online sensing and information retrieval}

Along with the evolution of the Internet, the information contributed publicly by all of us keeps increasing substantially. Through popular websites and social platforms such as Facebook, Twitter, Wikipedia, eventful.com Foursquare, etc., it is possible nowadays to collect information about popular events that happened in the past as well as information for those planned in the near future. The anticipation of the consequences of popular events in the transport system will allow us to better prepare for such scenarios.

Based on the spatio-temporal information that defines a special event, such as its title (what), starting and ending time (when), and venue (where), queries can be automatically constructed for retrieving relevant documents using web search engine Application Programming Interfaces (APIs). However, the construction of such questions or queries is a nontrivial task [27], because it contains a high risk of getting very broad matches. One trivial solution would be to just constrain to a limited number of online event announcement websites. But doing so risk to miss important information that could be elsewhere (e.g. venues own homepages, social media comments).

Another challenge is the fact that most of the retrieved documents contain details about an event in unstructured form and in a limited volume [28]. At the same time, many of the documents may be irrelevant to the matter of interest. Therefore, scoring metrics that determine the rank of each document with respect to many deciding factors have been developed and widely used. Google, for instance, indicates that they presently use over 200 factors to determine the ranking of the presented search results. 
The most common metric for text ranking is based on weighting the importance of a term in a document, based on the statistics of occurrence of the term. The "Term FrequencyInverse Document Frequency" (TF-IDF) is one of the most popular term-weighting schemes, that is also used in this research [29]. If correlates to the term's frequency, defined as the number of times term $t$ appears in the currently scored document d. Documents with higher score have more occurrences of a given term compared to the rest.

For the scores calculation, each set of documents is firstly represented in numerical form as a vector:

$$
d_{j}=\left(t_{1, j}, t_{2, j}, \ldots, t_{n, j}\right)
$$

where $t_{n, j}$ corresponds to a separate term in the document $j$ (also known as statespace model). If a term occurs in the document, its value in the vector is non-zero. If we denote the raw count by $f_{t, d}$, then the simplest $t f$ scheme is $t f(t, d)=f_{t, d}$. Other scheme possibilities from the literature are the Boolean frequency, where $t f(t, d)=1$ if $\mathrm{t}$ occurs in $\mathrm{d}$ and 0 otherwise, the logarithmically scaled frequency and the augmented frequency. In this research the latter is preferred, as it prevents a bias towards longer documents. The raw frequency is divided by the raw frequency of the most occurring term in the document:

$$
t f(t, d)=0.5+0.5 \cdot \frac{f_{t, d}}{\max \left\{f_{t^{\prime}, d}: t^{\prime} \in d\right\}}
$$

where $t^{\prime}$ represents the most occurring term.

The inverse document frequency $i d f$ is a measure of how much information the word provides. This value correlates to the inverse of the number of documents in which the term $\mathrm{t}$ appears. Its default computation is:

$$
i d f(t, D)=\log \frac{N}{|\{d \in D: t \in d\}|}
$$

where $\mathrm{N}$ is the total amount of documents in the corpus. The denominator represents the number of documents where the term $t$ appears.

Finally, the tf-idf is calculated as:

$$
t f i d f(t, d, D)=t f(t, d) \cdot i d f(t, D)
$$

The calculated weights tend to filter out common terms, because a high tf-idf score is reached by a high term frequency in the given document and a low document frequency of the term in the whole collection of documents. Having therefore performed all the above steps, we can successfully detect documents' significant words and efficiently expand our new queries in the second stage.

The goal of query expansion is to ultimately arrive to an "ideal query vector", that gives us the best possible, ranked, set of documents. We start with the basic query, and refine it with each new result. Assuming we have an oracle for relevance of a retrieved document (i.e. a mechanism that tells which documents are relevant from the list), we can incorporate 
this information using the method proposed by Rocchio in 1971, therefore known as Rocchio algorithm [30]:

$$
\overrightarrow{q_{m}}=\alpha \overrightarrow{q_{0}}+\beta \frac{1}{\left|D_{r}\right|} \sum_{\overrightarrow{d_{j}} \in D_{r}} \overrightarrow{d_{j}}-\gamma \frac{1}{\left|D_{n r}\right|} \sum_{\overrightarrow{d_{j}} \in D_{n r}} \overrightarrow{d_{j}}
$$

where $\overrightarrow{q_{0}}$ represents the original query, $D_{r}$ the set of relevant documents and $D_{n r}$ the set of irrelevant documents. All terms can be weighted separately in their respective document sets, using e.g. the TF-IDF statistic. The weights $\alpha, \beta$, and $\gamma$ are used to steer the importance of each set of documents. Some systems also ignore negative feedback $(\gamma=0)$ or leave the initial query part $\overrightarrow{q_{0}}$ unaltered, so that it is not taken into consideration in the equation. With the equation (5) updated questions can be formed for the web search engines, thus increasing the relevance of newly retrieved documents.

\subsection{Topic Modeling}

A considerable amount of important information about a planned event is in textual form. Adding to other structured information, such as date, time and location, we can find useful details concerning its content in the description, title or comments on the website hosting the announcement. To obtain an automated system, we still need to convert such data into a proper representation that a machine learning algorithm can understand. However, the dimensionality of the machine learning model will be increased beyond reasonable if we explicitly include the text, word by word. Natural language is rich in synonymy and polysemy, different announcers and locations may use different words, besides it is not always obvious which words are more "relevant". Topic modeling is the research topic that focuses on covering these weaknesses.

The approach of topic modeling is to represent a text document as a finite set of topics. These topics correspond to sets of words that tend to co-occur together rather than a single word associated with a specific topic. For example, a rock festival textual description could have a weight $w_{1}$ assigned to topic 1 (e.g. words related to concerts in general), $w_{2}$ of topic 2 (e.g. words related to festivals), $w_{3}$ of topic 3 (e.g. words related to the venue descriptions) and so on. In particular, we use a specific technique that is called Latent Dirichlet Allocation (LDA). For the readers that are familiar with Principal Components Analysis (PCA), there is a simple analogy: PCA re-represents a signal as a linear combination of its eigenvectors, while LDA re-represents a text as a linear combination of topics. In this way, we reduce the dimensionality from the total number of different words of a text to the number of topics, typically very low. Each document is represented as a distribution over topics, and each topic is a distribution over words. For further details concerning LDA's generative process please refer to the original article of David Blei and colleagues [31].

In the particular domain of urban computing, Pereira et al. [2] studied the problem of using event data to help predict public transport demand. Their approach consists of using LDA to learn a topic model, and using the topic assignments as features in probabilistic graphical model. Other recent research efforts have focused on tools dealing with event identification in real time using micro-blog services. Kireyev et al. [32] suggest that the 
family of topics models is a particularly promising tool for disaster response agencies, as Twitter often provides critical up-to-date and on-location updates about an unfolding crisis. Ramage et al. [33] present a partially supervised learning model (Labeled LDA) that maps the content of the twitter feed into different dimensions, including substance, style, status, and social characteristics of posts. Ma H. et al. [34] present a scalable implementation of a topic modeling (Adaptive Link-IPLSA) based method for online event analysis, which summarize the gist of the massive amount of changing tweets. All these studies further confirm the feasibility of topic modeling for microblog representation.

After finishing the task of finding the most representative topics of available event data [2], tweets [32, 33, 34], mobile Web usage logs [35], etc., follows the classification stage, where algorithms such as the linear Support Vector Machines (SVMs) categorize available records into the classes that we are interested in. The integration of the mechanism behind the max-margin prediction models (e.g., SVMs) with the mechanism behind the hierarchical Bayesian topic models (e.g., LDA) under a unified constrained optimization framework can be also implemented using the maximum entropy discrimination latent Dirichlet allocation (MedLDA) model [5]. While LDA is unsupervised and just gives us the topics that best represent the corpus of documents, MedLDA looks for the topics that maximize the performance of a certain classification task. Such topics may in fact be quite poor to represent the corpus of documents itself, but they are better to solve a certain classification task. Intuitively speaking, MedLDA tries to find the topics that enable the maximum possible margin between the classes in question. As a consequence, in our case, topics will either strongly support or strongly oppose the likelihood of an event to be a hotspot or not.

The classification rule for a given document is determined by:

$$
c^{*}=\operatorname{argmax} \mathbb{E}\left[\eta_{c}^{T} \bar{z} \mid \alpha, \beta\right]
$$

where $c^{*}$ is the predicted class, $\bar{z}$ is a vector with the topic proportions of the document, $\eta_{c}$ is a class-specific set of weights, $\alpha$ is a prior hyperparameter for the LDA component of the model, and $\beta$ contains $\mathrm{K}$ vectors, $\beta_{k}$, each one being the word distribution for the topic k. The estimation $\eta_{c}$ and $\bar{z}$, together with the best values of $\alpha$ and $\beta$ is accomplished using an Expectation-Maximization algorithm (EM). In the E-step, we assume $\alpha$ and $\beta$ to be fixed and estimate the posterior distributions for $\eta_{c}$ and $\bar{z}$; in the M-step, we use those distributions to estimate the best $\alpha$ and $\beta$. The process is repeated until convergence. For further details please refer to [5].

\section{Data Description and Preparation}

In the context of this research, two main categories of datasets were prepared and analyzed. The first category corresponds to taxi data, which are distributed by technology providers under the Taxicab \& Livery Passenger Enhancement Programs (TPEP/LPEP) and were made publicly available by the NYC Taxi and Limousine Commission (TLC) [36]. The raw dataset includes fields capturing pick-up and drop-off dates/times, pickup and dropoff locations, trip distances, itemized fares, rate types, payment types, and driver-reported passenger counts. 
The second category corresponds to event data, obtained from event listing sites, such as Facebook, Timeoutworld, Eventful and Ticketmaster. They were extracted automatically from the Web using either screen scraping techniques or API's. Table 1 shows an example of all event attributes collected using Facebooks API. A prerequisite for including an identified event in our database is the correct knowledge of its start time, its location as well as the title of the event. Very important elements of an event record were also considered its detailed description and the number of people attending the event (using mainly Facebooks API). However, it was not feasible to have the last two features in all our records.

Table 1: Event data excerpt.

\begin{tabular}{ll}
\hline name & example \\
\hline sid & facebook_235808830094292 \\
title & ZHU - Neon City Tour: Terminal 5 - New York, N... \\
venue & TERMINAL 5 \\
description & In an ever shifting social landscape of elusiv... \\
latitude & 40.769615 \\
longitude & -73.992770 \\
start & $2016-05-12$ 21:00:00 \\
end & $2016-05-12$ 23:00:00 \\
url $^{*}$ & https://www.facebook.com/events/235808830094292 \\
attending $^{*}$ & 978 \\
category $^{*}$ & MUSIC \\
address $^{*}$ & 610 W 56th St, New York, NY 10019, USA \\
\hline
\end{tabular}

\subsection{Hotspots identification using Kernel Density maps}

Special events, protests, visits by politicians, and so on attract many people in a certain area for a short or a long time period. A theater, a stadium, or an exhibition center emerges as a point of interest for many citizens; therefore, these locations influence the overall heat map of a city's daily trips. For the detection of unusual motions or interactions (hotspots), it is necessary to build representations of the selected areas of interest that are regions where the number of trips has significantly changed.

A general non-parametric technique that estimates the underlying density of historical taxi data, thereby avoiding the need to store the complete data, is kernel density estimation (KDE). Given a sample $S=x_{i i=1, \ldots, N}$ from a distribution with density function $p(x)$, an estimate $\hat{p}(x)$ of the density at $x$ can be calculated by using:

$$
\hat{p}(x)=\frac{1}{N} \sum_{i=1}^{N} K_{\sigma}\left(x-x_{i}\right)
$$

where $K_{\sigma}$ is a kernel function with a bandwidth (scale) $\sigma$ such as $K_{\sigma}(x)=\frac{1}{\sigma} K\left(\frac{x}{\sigma}\right)$. It is non-negative, integrates to one, and has a mean of zero. 
The kernel density values are represented as a two-dimensional array. For the Manhattan area, which is our main study area, a grid step was chosen with a value of 100 , which results in a space of approximately 93 meters on the longitudinal axis and 200 meters on the latitudinal axis between each grid point. The selected grid size gives us a fairly informative picture of taxi demand in the area of interest, since we believe that a popular venue can easily influence the demand conditions at a distance of up to 200 meters around it.

We calculated the kernel density values of an average day for the first six months of 2016 (six different heat maps, one for each month). At a later stage, we compared each day with the average demand day of the corresponding month. Some example heat maps are depicted in Figure 1. We standardized the final output using the Z-score formula:

$$
Z_{\text {diff }, i}=\frac{\hat{p}\left(x_{i}\right)-\hat{p}(x)}{\sigma}
$$

where $i$ is the examined day and $\sigma$ is the standard deviation of the average day. Through the Z-score data transformation, each kernel density value is given in units of how many standard deviations it is from the mean value and, consequently, how far it is from the demand levels of the average day. Z-score values above 2 will also emphasize the areas where a hotspot has been located and should be taken into account in the next stages of our analysis.

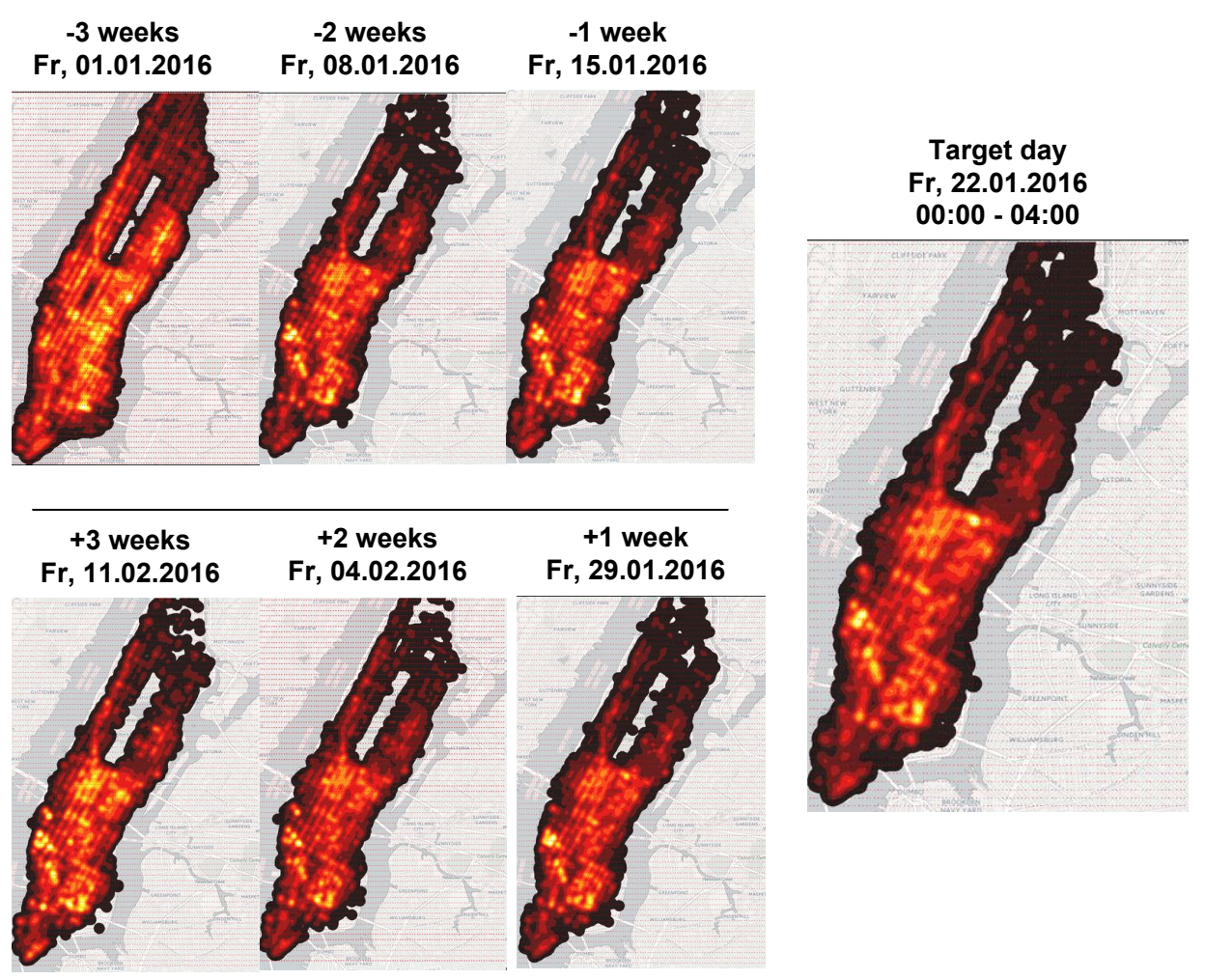

Figure 1: KDE of taxi pickups several weeks from each other. 
For more details on the average and individual day kernel density analysis, please refer to $[24]$.

Our objective is to develop an architecture that is able to classify potential hotspots for future events. An approximate average value for an event duration could be four hours, based on the existing list of events with a clear start and end time that we have at our disposal. Therefore, the concluding output of this stage is 6 heat maps per day so that our final Kernel density maps, and subsequently the located hotspots (Z-score value $>2$ ) on each one of them, correspond to a 4-hour time window. A characteristic example of a heat map with multiple events ending at the same time is depicted in Figure 2. The white "x"s define the events that took place on a Thursday in May. Through those heat-maps we are in a position to better visualize and evaluate the observed demand fluctuations. The number of taxi journeys recorded daily is enormous, so through the kernel density analysis we are able to make clearer results in the appropriate spatial resolution and avoid the chaotic depiction of thousands of points on the map.

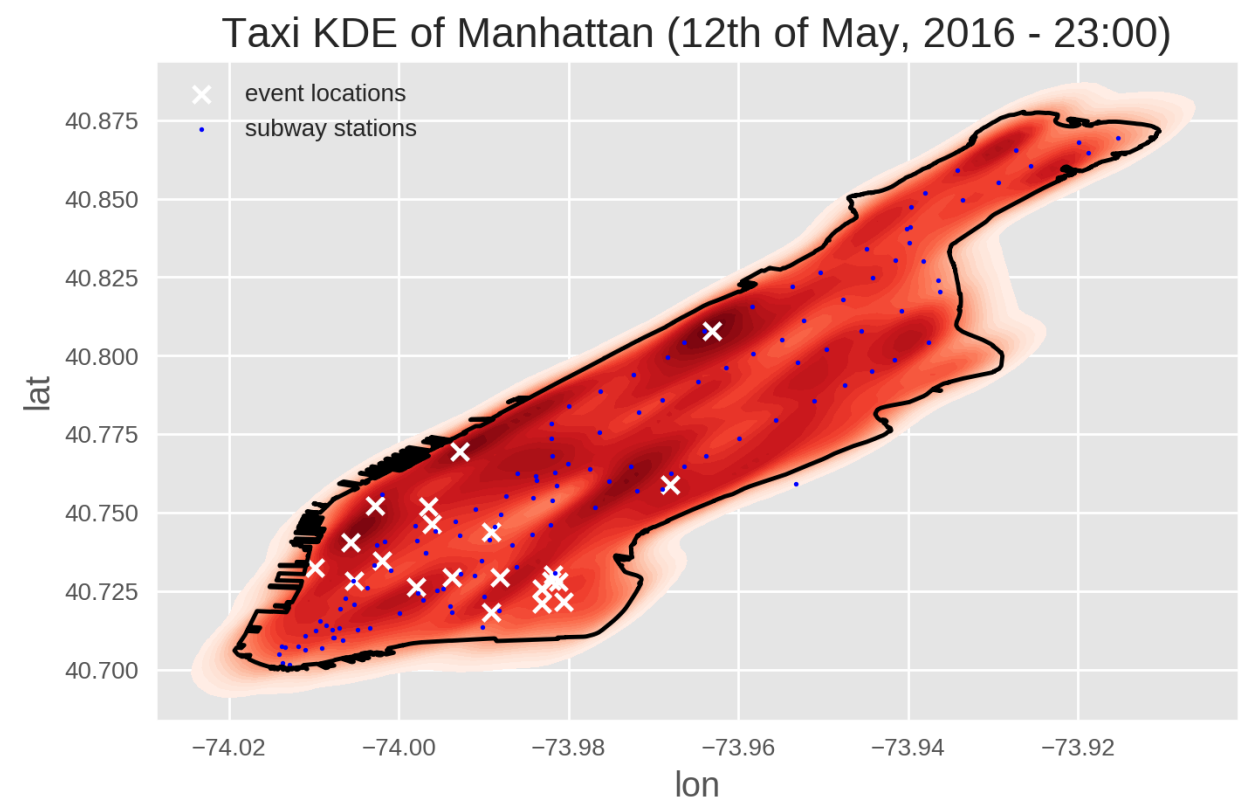

Figure 2: Heat map of taxi pickups in Manhattan

\subsection{Event records filtering}

The event database is a list of event titles, locations, start times and occasionally short descriptions. Since our study analysis is limited to the time period January - June 2016 and each heat map gives us possible hotspots during a 4-hour window, we:

- Discard events without end time information.

- Discard events without description. At a later stage, this information is necessary as it will be used for each event's attribute "category" (e.g. music, sports) determination. 
- Remove events where the venue is specified as "online" or "New York."

- Discard duplicates after records collapse. Their identification was mainly accomplished through the intersection identification of similar tokens in title and description.

At a second stage, event records are also correlated with the Z-score values of the corresponding heat maps. As described earlier, for each cell of the grid we have a Z-score value. Knowing the exact coordinates of each cell, as well as the event location, attempts were made to identify areas with a high Z-score within a radius of $300 \mathrm{~m}$ around the event.

After all, only events with an attending count over 500 people or a Z-score above " 2 " were kept for further exploration. Of all 36000 events that our initial database has, only 858 events met these criteria. High Z-Score values showed only 279 events, therefore the number of "abnormal" events is smaller compared to those events that did not affect transport system's balance considerably. The attributes of those 858 events are going to be used for training and testing the proposed methodology described in the following section.

\section{Methodology}

From a previous study [24] it is already known that there are several categories of events that can significantly affect the equilibrium of demand-supply of a transportation system. The degree of influence varies according to the day and time that the event takes place, its location, and of course its popularity. In this research, we are interested in predicting a demand hotspot considering the degree of influence of past events. Through machine learning techniques, several queries generation and expansion practices we will examine the prediction performance, of a classifier that determines whether an upcoming event is a hotspot or not.

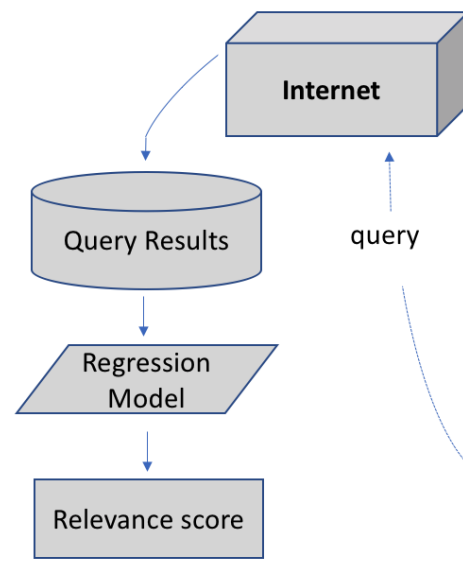

(a)

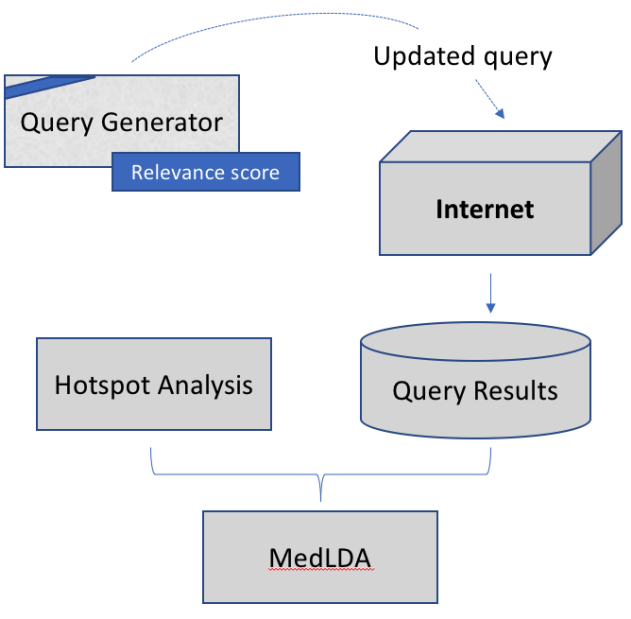

(b)

Figure 3: Methodology architecture 
The methodology presented in Figure 3 was developed to achieve the above objectives. It depicts the training stage which consists of two parts, applied in sequence: (a) the supervised regression model that will be responsible for characterizing texts as relevant or not, an essential component in the query expansion process (see Rocchio's formulation, eq. 5); and (b) the topic model that groups all retrieved documents based on specific events and gives us the word terms that increase hotspot's identification accuracy.

\subsection{Ranking framework development}

For the first part of the training mode, a detailed framework is formulated on the calculation of the relevance score between each event and the corresponding documents retrieved from the Web using search queries. The final scores will allow us to perform query expansion at a later stage.

Web search engines allow to ask questions in the form of queries to hopefully get some relevant information. In this study, the events database of 858 records is used for the query generation step. Three query sets were developed (see also Fig. 4):

- Query-Set A - Baseline structure : Only one query per event using the attributes "title" and "venue".

- Query-Set B - Varied structure : It includes three different queries per event using the attributes "start time" and "venue". The first query involves an unrestricted search of the terms, while the second and third query seeks exact matches of the titles using quoted phrases.

- Query-Set C - Enhanced structure : That set is formulated after the exploitation of Query-Set A and B and the receiving of the corresponding Web documents; for this and the characterization "enhanced". Our final Query-Set includes only one query per event using the attributes "start time" and "venue" and two expansion terms from the Query Set B.

Although there are plenty of the other possibilities, like extracted named entities from the event description, we assume that the selected attributes are the most informative for the event and its context. We also removed non-alphanumeric symbols from queries and added the phrase "New York" to narrow down the search results.

All queries in the respective sets are sent through different Searx instances to the web search engines Bing and Google and an aggregated list of search results is returned per query. The total number of retrieved documents per query is restricted to 50 for the first and third query set and to 30 for the second query set.

The Query-Set B - Varied structure was formed to be particularly restrictive. As mentioned earlier, it includes 3 different queries and their rules are further explained below:

- $1^{\text {st }}$ query: Exact match search of venue name and start time using quotes.

- $2^{\text {nd }}$ query: Exact match search of only the venue name. The date was not restricted. Three letter abbreviations of weekday and month were also added. 


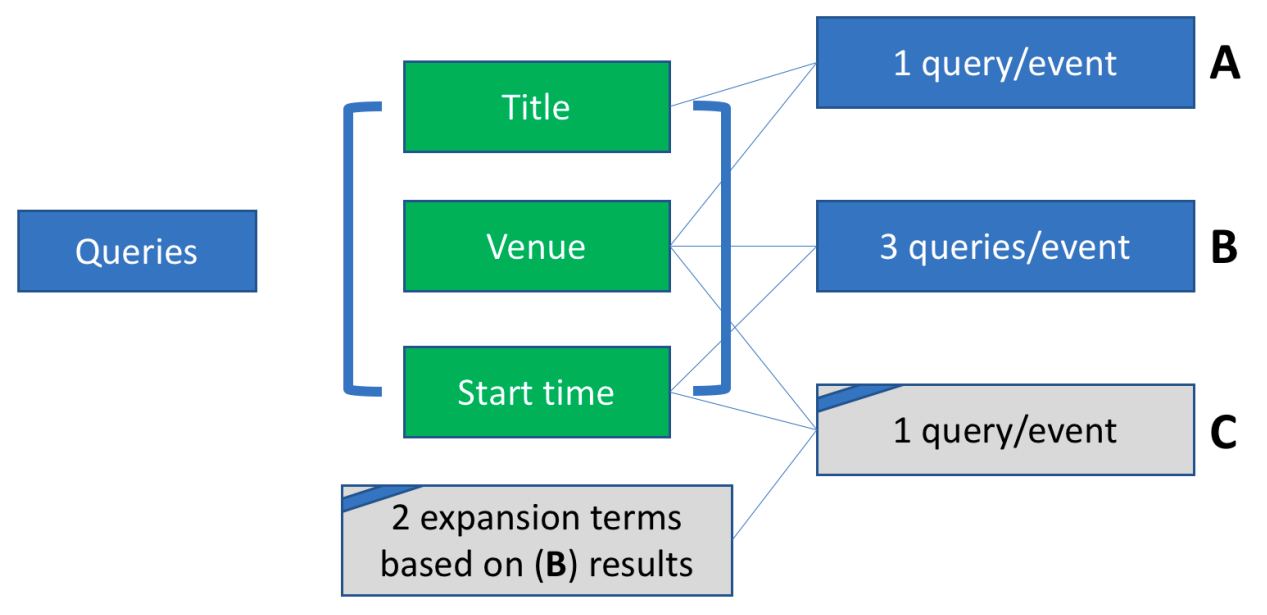

Figure 4: Methodology diagram for queries generation

- $3^{\text {rd }}$ query: Venue and start time are included without restrictions. Time is inserted after 12-hour time convention.

All response attributes from our queries are listed in Table 2. The shown example is returned using the $3^{\text {rd }}$ query of Query-Set B. It is worth mentioning that the retrieved URL can be used for document aggregation as it can only refer to a unique web page.

Table 2: Searx data excerpt of one query result.

\begin{tabular}{ll}
\hline name & example \\
\hline title & ZHU - Tickets - Terminal 5 - New York, NY - May 12th, 2016 \\
url & http://www.terminal5nyc.com/event/1107229-zhu-new-york/ \\
snippet & May 12, 2016 ... With sold out debuts in Los Angeles and New York City [...] \\
positions & {$[1]$} \\
score & 1 \\
engines & [google] \\
parsed_url & {$[$ "http", "www.terminal5nyc.com", "/event/1107229-zhu-new-york/"] } \\
queries & {$[3]$} \\
\hline
\end{tabular}

\subsection{Framework enhancement using event's category}

An attribute that we considered fairly important for a decent performance of our classifier, is the "category" of the event. With this term we denote the type of event that each database entry represents, e.g. music concert, sport game, demonstration, etc. This important feature is not feasible to always retrieve using APIs, so in many records this information was missing. Therefore, we applied the Term Frequency Inverse Document 
Frequency (TF-IDF) representation on the attribute "event description" to create an event category classifier.

According to J. Ramos et al. [37], TF-IDF calculates values for each word in a document through an inverse proportion of the frequency of the word in a particular document to the percentage of documents the word appears in. Words with high TF-IDF numbers imply a strong relationship with the document they appear in, suggesting that if that word were to appear in a query, the document could be of interest to the user. In our case, using the description of the event we will try to find the key words that have the highest weights and are highly correlated with our predefined categories. Before the calculation of TF-IDF weights, stopwords and terms whose frequency in documents exceeds $50 \%$ are removed. We have also developed a list of all possible event categories encoded as ordinal numbers. After TF-IDF implementation, the final weight matrix and the event categories were used as inputs for our stochastic gradient descent (SGD) classifier. The specific model is chosen because it is suited for handling sparse data with ease. From the initial dataset, $20 \%$ of events had the "category" attribute empty, and using the above tools, we managed to associate $75 \%$ of our event records with one of listed event categories. Table 3 summarizes the results of the classifier for 14 indicative categories, as well as its average performance for all included categories.

Table 3: SGD Classifier Performance

\begin{tabular}{|l|c|c|c|}
\hline Category & Precision & Recall & F1-Score \\
\hline Art & 0.76 & 0.84 & 0.80 \\
\hline Book & 0.86 & 0.83 & 0.85 \\
\hline Business & 0.74 & 0.78 & 0.76 \\
\hline Comedy & 0.94 & 0.88 & 0.91 \\
\hline Community & 0.82 & 0.68 & 0.74 \\
\hline Education & 0.74 & 0.71 & 0.72 \\
\hline Fitness & 0.82 & 0.79 & 0.81 \\
\hline Food & 0.80 & 0.81 & 0.80 \\
\hline Hobby & 0.83 & 0.75 & 0.79 \\
\hline Movie & 0.85 & 0.80 & 0.82 \\
\hline Music & 0.89 & 0.93 & 0.91 \\
\hline Nightlife & 0.84 & 0.87 & 0.85 \\
\hline Religion & 0.74 & 0.81 & 0.77 \\
\hline Volunteering & 0.72 & 0.64 & 0.68 \\
\hline \hline Average scores & 0.82 & 0.83 & 0.82 \\
\hline
\end{tabular}

\subsection{Relevance score estimation}

For the estimation of the relevance score, we will create a database of relevant documents for the target events. The criteria that we took into consideration for the identification of those documents are: 
- Direct matches on time information, and URLs

- String distance measurements

- Keywords matching

For the first criterion, the scores are based on the elements presented in Table 4. Great emphasis is given to the duration of the event, because those matches are rare yet very useful.

The second criterion implies the identification of similarity between two specified string groups. Four distance algorithms are used for that purpose: Levenshtein $l$, Ratcliff/Obershelp $r$, Sørensen/Dice $d$ and Jaccard $j$. The end return of each one of them is a value between 0 and 1. All four algorithms were applied in the title and description attributes of event records and document contents respectively. Then, each score is aggregated and to the final matching score calculation using the equation: $s=\frac{2(l+r+d+j)_{t i t l e}+(l+r+d+j)_{d e s c}}{2}$. Double weight was given to the "title" string group as it contains more relevant information about the event. The highest possible score on complete accordance of all groups has therefore chosen to be "6".

Table 4: Relevance Score Estimation

\begin{tabular}{|c|c|c|}
\hline Event Attribute & Document Content & Score \\
\hline \hline Date & Date & +0.25 \\
\hline Start Time & Start Time & +0.25 \\
\hline End Time & End Time & +0.25 \\
\hline Duration & Duration & +1 \\
\hline URL & URL & +2 \\
\hline Domain Name & Domain Name & +1 \\
\hline
\end{tabular}

The final criterion for the relevance score calculation is the keywords matching. Exact keyword matches will increase our relevance score by 2 , since it can be considered a relatively strong indicator.The Rapid Automatic Keyword Extraction (RAKE) algorithm [38], an unsupervised, domain-independent, and language-independent method for extracting keywords from individual documents was used for each text. It is also worth noting that, since the keyword extraction only happens on equally long snippets, there is no bias towards larger texts. Finally, besides the exact keyword matching identification, we also considered word similarities. When very similar keywords are detected using the Ratcliff/Obershelp similarity measurement $(r>0.85)$, the total relevance score increases by 1 .

The threshold value between relevant and non-relevant documents discrimination was the score "6". In other scenarios, when more queries are used, this score can be adjusted accordingly. Of the 858 events, 717 were associated with at least one relevant retrieved document. For the rest 141 events, it is interesting to note that 36\% (52) of them were correlated with a demand hotspot at a previous stage (see subsection 3.2) and we were 
expecting to find effortlessly relevant documents, since they looked fairly popular only by the heat-map inspection.

Thereafter follows the query expansion stage. Using the the Rocchio algorithm that was presented in section 2, updated queries were formulated with the venue name, the start time and two new expansion terms. The $3^{\text {rd }}$ Query from the $2^{\text {nd }}$ Query Set was used as the initial base query $q_{0}$ and the numerical statistic TF-IDF was applied as a weighting scheme for the respective document set with $\beta=0.75$ and $\gamma=0.15$. Terms that are finally chosen to be used in the new query, but already exist in the base query $q_{0}$ terms, are ultimately rejected. The first 50 documents that the web search engine returns using the expanded query are then saved for the supervised classification model training and Query Sets' evaluation and comparison.

\subsection{Supervised Topic Modeling algorithm Application}

The performance of our final model is not only based on the Query Sets' application but also on the selection of the classification mechanism and its parameters. MedLDA has several hyperparameters to tune, namely $\alpha, l$ (penalty term for misclassifications) and $C$ (penalty for soft margin slack variables). Following the results of a prior 10-fold cross-validation grid search methodology implementation [39], we set $\alpha=0.001 / K, l=10$, and $C=30$. The topic number $K$ was also set to 10, based on the same previous research.

Apart from the input constants mentioned above, MedLDA receives as input the retrieved documents from our search queries. All event documents were placed in chronological order since they are not independent and identically distributed. The same keywords presented in the training set, were also used in the test set.

As mentioned in 3.2, in total 279 events met the hotspot criteria from the whole dataset of 878 events. Subsequently, our dataset is imbalanced, since the classification categories are not approximately equally represented. Therefore, under-sampling of the majority (normal) class has been implemented as a good means of increasing the sensitivity of a classifier to the minority class (hotspots). Using the synthetic minority over-sampling technique (SMOTE), the correct equilibrium of hotspot-correlated events between training and test set was achieved.

We will compare MedLDA's performance with three other baseline models, all based on a typical two-stage process that firstly determines the topics using the standard unsupervised LDA, and secondly characterizes each event as a demand hotspot or not using a classifier. The classifiers that we chose are the Support Vector Machine (SVM) classifier, because MedLDA integrates the same mechanism behind the max-margin prediction, Logistic Regression (LR) and the Multi-layer Perceptron (MPL) classifier, as two very popular benchmark algorithms. The output of all these frameworks is the prediction of demand hotspots' presence, based on the characteristics of each event, as represented through the topics.

The comparison will be based on three measures: accuracy, F1-score, standard deviation $(\sigma)$ and Cohens kappa statistic $(\kappa)$. Since the accuracy is just the percentage of correctly classified instances, it could be misleading because the test set is imbalanced. Therefore, the F1-score is been taken into consideration as it relates the number of correctly classified 
positive instances with the quantity of the missed ones. Furthermore, $\kappa$ quantifies the chances that the agreement between the model's results and the perfect classifier is random. The value of $\kappa=0$ corresponds to the performance of a random classifier, while $\kappa=1$ corresponds to the perfect classification without random coincidence. Finally, the standard deviation was also included as an additional accuracy indicator.

\section{Results}

The final results of MedLDA, LDA+SVM, LDA+LR and LDA+MLP choosing $K=10$ as the preferred number of topics, are presented in Table 6. It can be concluded that the performance of MedLDA is much better than all the other approaches. It is also worth noting that there is no noticeable performance difference between the three Query sets. Extracted topics using the aggregated documents of Query-Set B were as useful as the ones extracted from the baseline queries for hotspot prediction.

For a more detailed analysis of the results, the most discriminative terms of each topic as well as their assigned $\eta$ are illustrated in Fig. 5. Table 5 additionally summarizes the number of hotspots and scheduled events that are correlated with twelve of the most popular venues in NYC. Looking thoroughly the Query-Set A (Fig. 5), the term "Hirschfeld" appears on the top topic (10a). The specific topic has also a high $\eta$ value $(\eta=12.45)$ which indicates that the Hirschfeld Theater, which is located in midtown Manhattan, is very often associated with a hotspot presence. More generally, most of the stemmed terms in this query set can be separated into the following abstract classes: (i) the type of venue (e.g. "restaurant" in topic 7a and "gallery" in topic 10a), (ii) artist name or performance title words (e.g. the name "eric prydz" of a musician in topic $2 \mathrm{a}$ and the title of a theater performance: "waitress" in topic 5) and (iii) event categories (e.g. "music" in 2a and "family" in 6a).

Table 5: Unique event venues and their hotspots.

\begin{tabular}{lll}
\hline & hotspot counts & total event counts \\
\hline Central Park & 7 & 20 \\
TERMINAL 5 & 6 & 15 \\
SOBs Sounds of Brazil & 5 & 7 \\
Cornelia Street Cafe & 4 & 9 \\
Al Hirschfeld Theatre & 4 & 5 \\
Intrepid Sea Air and Space Museum & 4 & 5 \\
Le Bain at The Standard High Line & 4 & 5 \\
BB King Blues Club and Grill & 3 & 19 \\
City Winery New York & 0 & 24 \\
Madison Square Garden & 0 & 11 \\
Webster Hall & 0 & 11 \\
\hline
\end{tabular}

For the Query-Set C, a clear contribution to the hotspot classification seems to make the first (10b) and last topic (1b). The keywords "madison" and "garden" that appear in 


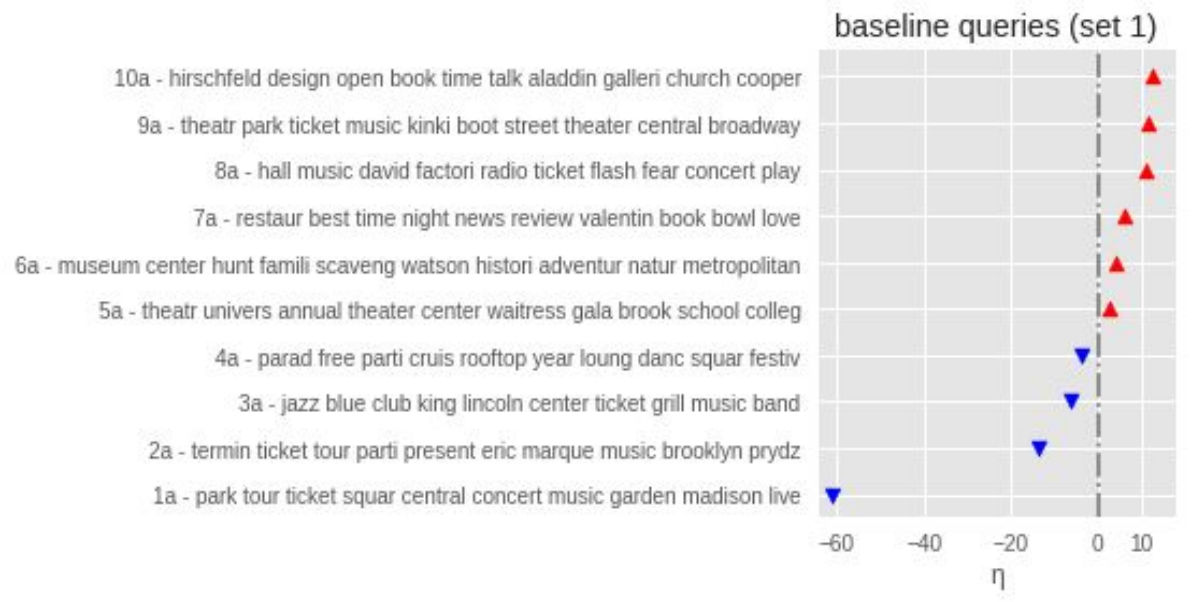

expanded queries (set 3)

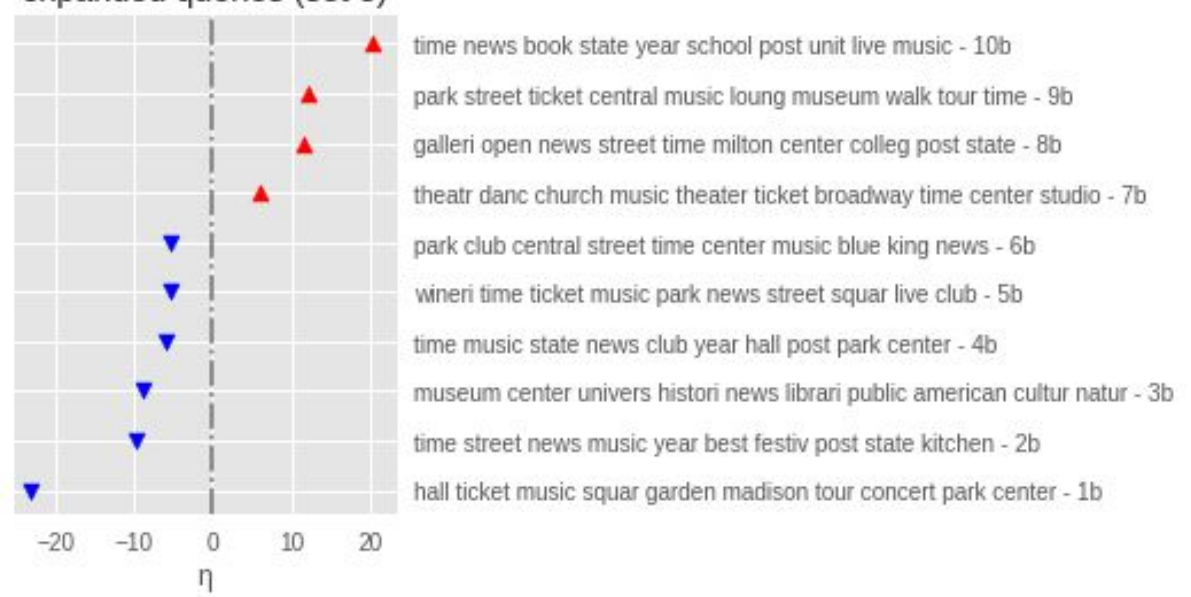

Figure 5: Topics for baseline queries with assigned $\eta$.

topic 1b (and 1a from the Query-Set A) give us a clear indication that special events in Madison Square Garden are not related to high taxi demand existence. This conclusion can be also justified by the fact that very good public transport services are offered in the vicinity, and there may be already a significant number of taxi demand regardless of events (notice that a hotspot is a relative measure, i.e. a strong deviation from the norm).

Other conclusions about the total performance of query sets are:

- The Query-Set $\mathbf{C}$ has more time-related keywords in its topics, since a different date format was used in its corresponding queries that was apparently more useful in searching relevant documents.

- The Query-Set C has a wider keyword variety, which allows for clearer classification results.

- Fund-raising events that took place in Central Park (e.g. "AIDS Walk" and "9/11 
Table 6: Comparison of classification performance of different Query sets.

\begin{tabular}{cccc}
\hline & Accuracy & $\mathrm{F}_{1}$-score & $\kappa$ \\
\hline LDA + SVM - Query-Set A & 0.501 & 0.525 & 0.141 \\
LDA + SVM - Query-Set B & 0.512 & 0.517 & 0.135 \\
LDA + SVM - Query-Set C & 0.622 & 0.564 & 0.265 \\
LDA + LR - Query-Set A & 0.535 & 0.497 & 0.126 \\
LDA + LR - Query-Set B & 0.559 & 0.501 & 0.203 \\
LDA + LR - Query-Set C & 0.600 & 0.532 & 0.214 \\
LDA + MLP - Query-Set A & 0.534 & 0.500 & 0.133 \\
LDA + MLP - Query-Set B & 0.494 & 0.485 & 0.088 \\
LDA + MLP - Query-Set C & 0.600 & 0.543 & 0.226 \\
MedLDA - Query-Set A & 0.715 & 0.484 & 0.296 \\
MedLDA - Query-Set B & 0.732 & 0.5 & 0.329 \\
MedLDA - Query-Set C & 0.756 & 0.618 & 0.439 \\
\hline
\end{tabular}

Memorial Walk and Run“), as well as street festivals that were very popular in June, are well represented by topic $9 \mathrm{~b}$.

- The Query-Set $\mathbf{C}$ has the highest rate of false positives, due to the fact that after the query expansion stage, the results returned by the search engine are not filtered, and documents with only time information and no accurate event correlation are included in the topic model.

- According to the proportions of event categories, there was an above average number of hotspot events in the category "nightlife" with over $30 \%$ of them ending at 4 a.m..

\section{Conclusion}

We proposed a framework for real-world phenomena dynamics prediction, and more specifically for taxi demand prediction during non-recurrent events. We combined information extracted from the Web with time-series data to build a predictive model of taxi demand hotspots around special event venue areas. This is typically a challenging case for transport planning since special events originate high variance in demand. Taxi demand is correlated with many parameters of underlying information and, currently, most taxi centers rely on formal processes and manual work for a fleet organization and taxi distribution. Even the more advanced new services, like Uber or Lyft, still face great challenges in terms of demand prediction. Our results show that information retrieval using query expansion methods outperforms other baseline methods that rely solely on the basic attributes of an event.

It is concluded that there are a lot of compromises that need to be made in real-world applications. A methodology that relies on commercial search engines has its shortcomings since there is limited access to their full search index. Structural data from transport services 
and event listing sites do not always contain enough details for an adequate correlation of different sources of information.

In future work, we aim at exploring at a deeper level the potentials of the proposed machine-learned ranking model. More complicated forms of query expansion frameworks will be examined and the contribution of events' descriptions will be considered, since the implemented analysis showed that they contain very important keywords.

\section{References}

[1] F. C. Pereira, F. Rodrigues, M. Ben-Akiva, Using data from the web to predict public transport arrivals under special events scenarios, Journal of Intelligent Transportation Systems 19 (3) (2015) 273-288.

[2] F. C. Pereira, F. Rodrigues, E. Polisciuc, M. Ben-Akiva, Why so many people? explaining nonhabitual transport overcrowding with internet data, IEEE Transactions on Intelligent Transportation Systems 16 (3) (2015) 1370-1379.

[3] I. Markou, F. Rodrigues, F. C. Pereira, Real-time taxi demand prediction using data from the web, in: ITSC, 2018, IEEE, 2018.

[4] F. Rodrigues, I. Markou, F. C. Pereira, Combining time-series and textual data for taxi demand prediction in event areas: a deep learning approach, Information Fusion 49 (2019) 120-129.

[5] J. Zhu, A. Ahmed, E. P. Xing, Medlda: maximum margin supervised topic models for regression and classification, in: Proceedings of the 26th annual international conference on machine learning, ACM, 2009, pp. 1257-1264.

[6] W. M. Dunn Jr, S. P. Latoski, E. Bedsole, Planned special events: Checklists for practitioners, Tech. rep. (2006).

[7] F. Coutroubas, N. Tzivelou, Public transport planning for the greatest event: the 2004 olympic games, in: Proceedings of the ETC, Strasbourg, France, 2003.

[8] C. Fuhs, P. Brinckerhoff, Synthesis of active traffic management experiences in europe and the united states, Tech. rep., United States. Federal Highway Administration (2010).

[9] A. Kuppam, R. Copperman, T. Rossi, V. Livshits, L. Vallabhaneni, T. Brown, K. DeBoer, Innovative methods for collecting data and for modeling travel related to special events, Transportation Research Record: Journal of the Transportation Research Board (2246) (2011) 24-31.

[10] S. Shahin, T. O. Hüseyin, Ö. S. Kemal, Evaluating transportation preferences for special events: A case study for a megacity, istanbul, Procedia-Social and Behavioral Sciences 111 (2014) 98-106.

[11] M.-S. Chang, P.-R. Lu, A multinomial logit model of mode and arrival time choices for planned special events, Journal of the Eastern Asia Society for Transportation Studies 10 (2013) 710-727.

[12] J. Yuan, Y. Zheng, L. Zhang, X. Xie, G. Sun, Where to find my next passenger, in: Proceedings of the 13th international conference on Ubiquitous computing, ACM, 2011, pp. 109-118.

[13] F. Rodrigues, M. Lourenco, B. Ribeiro, F. C. Pereira, Learning supervised topic models for classification and regression from crowds, IEEE transactions on pattern analysis and machine intelligence 39 (12) (2017) 2409-2422.

[14] J. Xu, R. Rahmatizadeh, L. Bölöni, D. Turgut, Real-time prediction of taxi demand using recurrent neural networks, IEEE Transactions on ITS.

[15] N. Davis, G. Raina, K. Jagannathan, A multi-level clustering approach for forecasting taxi travel demand, in: ITSC, 2016, IEEE, 2016, pp. 223-228.

[16] L. Moreira-Matias, J. Gama, M. Ferreira, J. Mendes-Moreira, L. Damas, Predicting taxi-passenger demand using streaming data, IEEE Transactions on Intelligent Transportation Systems 14 (3) (2013) 1393-1402.

[17] Y. Tong, Y. Chen, Z. Zhou, L. Chen, J. Wang, Q. Yang, J. Ye, W. Lv, The simpler the better: a unified approach to predicting original taxi demands based on large-scale online platforms, in: Proceedings of the 23rd ACM SIGKDD Intern. Conf. on Knowledge Discovery and Data Mining, ACM, 2017, pp. 1653-1662. 
[18] Y. Lv, Y. Duan, W. Kang, Z. Li, F.-Y. Wang, Traffic flow prediction with big data: a deep learning approach, IEEE Transactions on Intelligent Transportation Systems 16 (2) (2015) 865-873.

[19] X. Ma, Z. Tao, Y. Wang, H. Yu, Y. Wang, Long short-term memory neural network for traffic speed prediction using remote microwave sensor data, Transportation Research Part C: Emerging Technologies 54 (2015) 187-197.

[20] J. Zhang, Y. Zheng, D. Qi, Deep spatio-temporal residual networks for citywide crowd flows prediction., AAAI (2017) 1655-1661.

[21] E. F. Morgul, K. Ozbay, Revisiting labor supply of new york city taxi drivers: Empirical evidence from large-scale taxi data, in: TRB 94th Annual Meeting, no. 15-3331, 2015.

[22] C. Yang, E. J. Gonzales, Modeling taxi demand and supply in new york city using large-scale taxi gps data, in: Seeing Cities Through Big Data, Springer, 2017, pp. 405-425.

[23] K. Zhao, D. Khryashchev, J. Freire, C. Silva, H. Vo, Predicting taxi demand at high spatial resolution: Approaching the limit of predictability, in: 2016 IEEE Intern. Conf. on Big Data, IEEE, 2016, pp. 833-842.

[24] I. Markou, F. Rodrigues, F. C. Pereira, Use of taxi-trip data in analysis of demand patterns for detection and explanation of anomalies, Transportation Research Record: Journal of the Transportation Research Board (2643) (2017) 129-138.

[25] M. A. Yazici, C. Kamga, A. Singhal, A big data driven model for taxi drivers' airport pick-up decisions in new york city, in: 2013 IEEE Intern. Conf. on Big Data, IEEE, 2013, pp. 37-44.

[26] C. Kamga, M. A. Yazıcı, Temporal and weather related variation patterns of urban travel time: Considerations and caveats for value of travel time, value of variability, and mode choice studies, TR-C: Emerging Technologies 45 (2014) 4-16.

[27] C. Hölscher, G. Strube, Web search behavior of internet experts and newbies, Computer networks 33 (1-6) (2000) 337-346.

[28] H. Schütze, C. D. Manning, P. Raghavan, Introduction to information retrieval, Vol. 39, Cambridge University Press, 2008.

[29] K. S. Jones, A statistical interpretation of term specificity and its application in retrieval, Journal of Documentation 28 (1972) 11-21.

[30] J. J. Rocchio, Relevance feedback in information retrieval, in: G. Salton (Ed.), The Smart retrieval system - experiments in automatic document processing, Englewood Cliffs, NJ: Prentice-Hall, 1971, pp. 313-323.

[31] D. M. Blei, A. Y. Ng, M. I. Jordan, Latent dirichlet allocation, Journal of machine Learning research 3 (Jan) (2003) 993-1022.

[32] K. Kireyev, L. Palen, K. Anderson, Applications of topics models to analysis of disaster-related twitter data, in: NIPS Workshop on Applications for Topic Models: Text and Beyond, Vol. 1, Canada: Whistler, 2009.

[33] D. Ramage, S. T. Dumais, D. J. Liebling, Characterizing microblogs with topic models., ICWSM 10 (2010) $1-1$.

[34] H. Ma, B. Wang, N. Li, A novel online event analysis framework for micro-blog based on incremental topic modeling, in: Software Engineering, Artificial Intelligence, Networking and Parallel \& Distributed Computing (SNPD), 2012 13th ACIS International Conference on, IEEE, 2012, pp. 73-76.

[35] J. Yuan, F. Gao, Q. Ho, W. Dai, J. Wei, X. Zheng, E. P. Xing, T.-Y. Liu, W.-Y. Ma, Lightlda: Big topic models on modest computer clusters, in: Proceedings of the 24th International Conference on World Wide Web, International World Wide Web Conferences Steering Committee, 2015, pp. 1351-1361.

[36] TLC Trip Record Data, http://www.nyc.gov/html/tlc/html/about/trip_record_data.shtml, [Online; accessed 21-March-2018] (2018).

[37] J. Ramos, et al., Using tf-idf to determine word relevance in document queries, in: Proceedings of the first instructional conference on machine learning, Vol. 242, 2003, pp. 133-142.

[38] S. Rose, D. Engel, N. Cramer, W. Cowley, Automatic keyword extraction from individual documents, Text Mining: Applications and Theory (2010) 1-20.

[39] S. Borysov, M. Lourenço, F. Rodrigues, A. Balatsky, F. Pereira, Using internet search queries to 
predict human mobility in social events, in: Intelligent Transportation Systems (ITSC), 2016 IEEE 19th International Conference on, IEEE, 2016, pp. 1342-1347. 